\title{
A HIGH-FIDELITY AIRBUS BENCHMARK FOR SYSTEM FAULT DETECTION AND ISOLATION AND FLIGHT CONTROL LAW CLEARANCE
}

\section{Ph. Goupil and G. Puyou}

AIRBUS Operations SAS

316 Route de Bayonne, Toulouse 31060, France

\begin{abstract}
This paper presents a high-fidelity generic twin engine civil aircraft model developed by Airbus for advanced flight control system research. The main features of this benchmark are described to make the reader aware of the model complexity and representativeness. It is a complete representation including the nonlinear rigid-body aircraft model with a full set of control surfaces, actuator models, sensor models, flight control laws (FCL), and pilot inputs. Two applications of this benchmark in the framework of European projects are presented: FCL clearance using optimization and advanced fault detection and diagnosis (FDD).
\end{abstract}

\section{INTRODUCTION}

Realistic aircraft models are more and more needed for development and assessment of new flight control techniques. Worldwide manufacturers use several kinds of models and simulators, the complexity of which are adapted to the required level of design and validation. The state of practice is the following: on the one hand, roughly linear models are used for FCL design and first assessment. On the other hand, system and system components monitoring are widely used for ensuring the aircraft fault tolerance. They are developed taking into account system specification and system models and, by a large majority, are based on hardware redundancy. The whole system validation and verification proceeds through several steps [1]: tests on a desktop simulator coupled to a rigid aircraft model, tests on a System Integration Bench (a test bench with simulated inputs and observation of computer internal variables offering the possibility of validating degraded configurations like, e.g., low hydraulic pressure and high aerodynamic loads), tests on the "Iron Bird" (a bench that is a kind of very 
light aircraft, without the fuselage, the structure, the seats, etc., but with all system equipment installed and powered as on a real aircraft), and tests on a flight simulator (a test bench with a real aircraft cockpit, flight control computers, and coupled to a rigid aircraft model). The flight simulator can be coupled to the Iron Bird. Finally, flight tests are performed on several aircraft fitted with "heavy" flight test instrumentation. For transversal studies or for collaborative works involving the aircraft manufacturer, autonomous means and tools are needed (decoupled from in-house ones), enough representative but without full access to the manufacturer know-how. This is why simulation benchmarks are developed based on an aircraft model (see, for example, [2] where a Boeing model has been used for developing a benchmark dedicated to fault tolerant flight control scheme design, and additional reference [3]). This paper deals with the general description of an Airbus benchmark used for advanced Flight Control System development. In particular, the development of such benchmark was driven by the requirements of FCL clearance and FDD. It has been used in the FP6 European COFCLUO (Clearance Of Flight Control Laws Using Optimization) project [4] and is currently used in the FP7 European ADDSAFE project (Advanced Fault Diagnosis for Sustainable Flight Control and Guidance) [5].

The Airbus benchmark is a complete aircraft model, highly representative of a generic twin engine civil commercial aircraft including the nonlinear rigid-body aircraft model with a full set of control surfaces (rudder, elevators, Trimmable Horizontal Stabilizer, spoilers, ailerons), actuator models, sensor models, FCL and pilot inputs. The major issue that drives this model structure is the ability to perform a validation of requirements on the whole flight domain considering a wide class of pilot inputs and wind perturbations as well as sufficiently detailed actuator and sensor models for representative fault injection and diagnosis.

This paper is organized as follows: in section 2, the main features of each benchmark components will be underlined to show the model complexity and representativeness. Section 3 illustrates the use of the benchmark for FCL clearance in the frame of the FP6 European project COFCLUO. Section 4 exemplifies the benchmark as a tool for FDD in the frame of the FP7 European project ADDSAFE. Some concluding remarks and perspectives are finally given in section 5 .

\section{AIRBUS BENCHMARK DESCRIPTION}

The model is highly representative of the aircraft flight physics and handling qualities. It is a closed-loop nonlinear model based on the following representation (Fig. 1) with five main components: pilot inputs, FCL, actuators, flight 


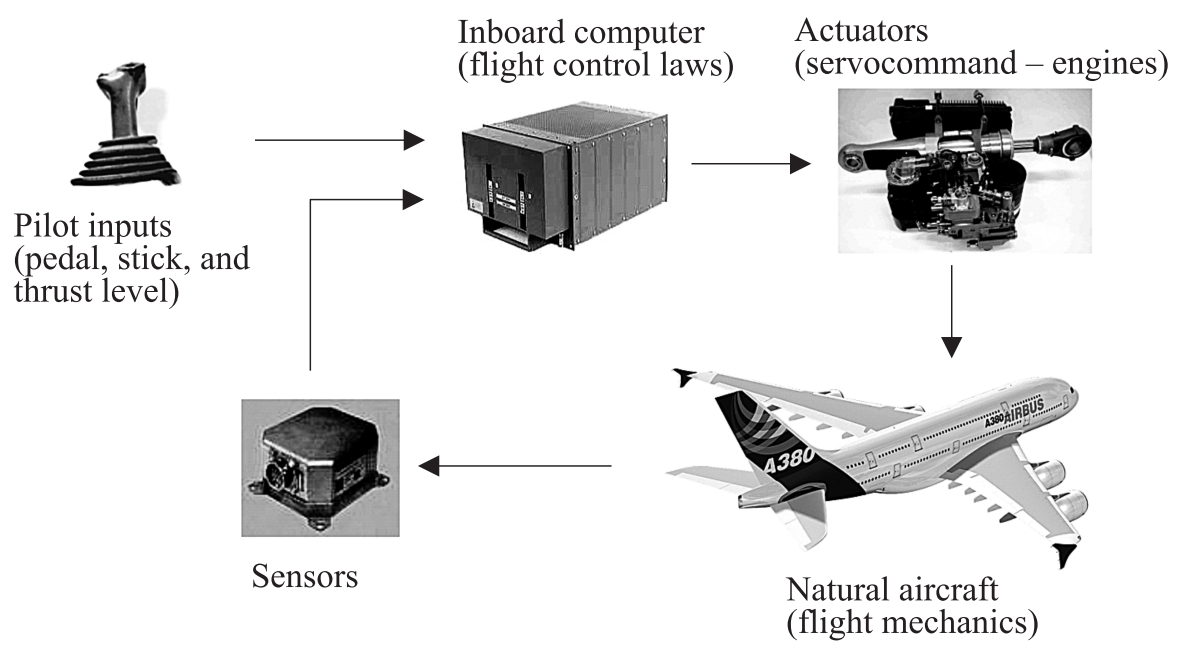

Figure 1 Airbus benchmark structure

mechanics, and sensors. It has been developed under the Matlab/Simulink environment and is usable under the same software thanks to dedicated Graphical User Interfaces (GUI). For a given flight scenario, the aircraft mass and center of gravity location should be chosen from the mass and balance domain. However, it should be noted that during the course of the chosen scenario, these parameters do not evolve (e.g., no fuel consumption model).

\subsection{Pilot Inputs}

The model is controlled through the following inputs: the side stick (longitudinal and lateral inputs), the pedals, the high-lift configuration lever (slats and flaps), the airbrakes, and the throttle lever. The side stick, pedal, and thrust inputs can either be defined by the user or independently managed by a pilot model which roughly corresponds to an enhanced set of autopilot hold modes. Each mode can be independently activated so that users can mix inputs provided by them and the pilot model.

\subsection{Actuator Modeling}

The actuator modeling is based on three elements: the actuator model itself, a control surface position saturation that could be dissymmetric and a rate limiter 
representing the physical limitations. The model input is the commanded actuator position (output of the FCL computation) while the output is the realized actuator position. Two kinds of models can be used depending on the benchmark use. If the goal is to focus on handling qualities evaluation (see section 3), then simple actuator models are sufficient (first- or second-order transfer functions). If system components fault detection is studied, then a more detailed model can be used. In this case, the actuator model describes the physical behavior of the actuator rod speed in function of the hydraulic pressure delivered to the actuator and in function of the forces applied on the control surface and reacted by the actuator [6]. It can be expressed by the following deterministic state-space model:

$$
\begin{aligned}
\dot{x}(t) & =f(u(t), x(t), \theta(t)) ; \\
y(t) & =x(t)
\end{aligned}
$$

where $f$ is the nonlinear function detailed in [6] and $\theta(t)=\left[\Delta P(t), F_{\text {aero }}(t) K_{a}(t)\right]$ is the vector of parameters representing, respectively, the hydraulic pressure delivered to the actuator, the aerodynamic forces applying on the control surface, and the damping coefficient associated to the adjacent passive actuator [6] (in case of two actuators per control surface in an active/passive scheme). Although it is termed an actuator model, it should be noted that the modeling covers the control loop between the Flight Control Computer and the control surface, including these two elements. This benchmark does not aim at studying the aircraft management after failure detection or abnormal configuration detection (automatic failure management so-called "reconfiguration"); so, only one actuator is simulated per control surface (no adjacent redundant actuator).

\subsection{Flight Mechanics}

Flight mechanics modeling is based on the so-called fundamental principle of dynamics. Both quaternion system and Euler angle formulations can be used. The main forces and moments acting on the aircraft are simulated: aerodynamic effects, gravity, and engine thrust. When using quaternions, the aircraft motion is described by

$$
\begin{aligned}
m \dot{\vec{V}} & =F_{a}+F_{g}+F_{p} ; \\
\dot{\bar{q}} & =\frac{1}{2} \bar{q} \otimes \Omega ; \\
I_{T} \dot{\Omega} & =M_{a_{G}}+M_{p_{G}}-\Omega \wedge I_{T} \Omega
\end{aligned}
$$

where $m$ is the mass; $\bar{V}$ is the inertia velocity vector; $F_{a}$ is the sum of the aerodynamic forces; $F_{g}$ is the sum of the gravity forces; $F_{p}$ is the sum of the 
engines forces; $\bar{q}$ is the quaternion vector describing attitude; $\Omega$ is the angular velocity vector in the aircraft coordinate system; $I_{T}$ is the inertial tensor; $M_{a_{G}}$ is the aerodynamic forces induced momentum at the center of gravity; and $M_{p_{G}}$ is the engine forces induced momentum at the center of gravity.

The main forces and moments acting on the aircraft are due to the aerodynamic effects $\left(F_{a}\right.$ and $\left.M_{a_{G}}\right)$, gravity $\left(F_{g}\right)$, and engine thrust $\left(F_{p}\right.$ and $\left.M_{p_{G}}\right)$. The main loads used in the flight mechanics equations are the aerodynamic loads. They are determined most often by identification of aerodynamic coefficients $\left(C_{b}, C_{m}, C_{n}, C_{x}, C_{y}\right.$, and $\left.C_{z}\right)$. In this benchmark, these global coefficients are expressed through neural network as nonlinear functions of inputs describing the state of the system such as velocities, altitude, attitude, external conditions, the configuration of the aircraft (slats, flaps, spoilers, etc.), and the position of control surfaces (ailerons, elevators, rudder, etc.). The mass of the aircraft is considered to be applied at its center of gravity along the vertical axis. Corresponding longitudinal and lateral forces are generated due to the attitude variation. Engine forces are modeled by the forces applied at each engine and directed along their own longitudinal axes. These axes are projected onto the aircraft coordinate system to determine the corresponding components (for more details, see $[4])$.

\subsection{Control Laws}

This current benchmark deals with manual control (so autopilot guidance laws are not included) but for better manoeuvre management, the autothrust control law, which is useful for managing the thrust and maintain the speed constant, is kept. Regarding manual laws, as the goal is not to study failure reconfiguration, all the unusual control laws are removed. Except for those points, all inboard computer implemented elements are kept. The control law module is a compiled black-box. The control laws mainly consist of proportional output feedback with an integral control error feedback to ensure zero steady-state tracking error. Their gains are scheduled to cover the whole flight domain.

\subsection{Sensors}

An integrated sensor model allows simulating very accurately all sensors involved. A plethora of information is needed and integrated in the model: sensor characteristics (location, noise, filter, etc.), calibration data, aerodynamic coefficients, flight mechanics equations, system requirements (e.g., delays), etc. This model is thus very complex and will not be detailed here. A management system has been implemented for simulating the redundant sensor choice and monitoring, 


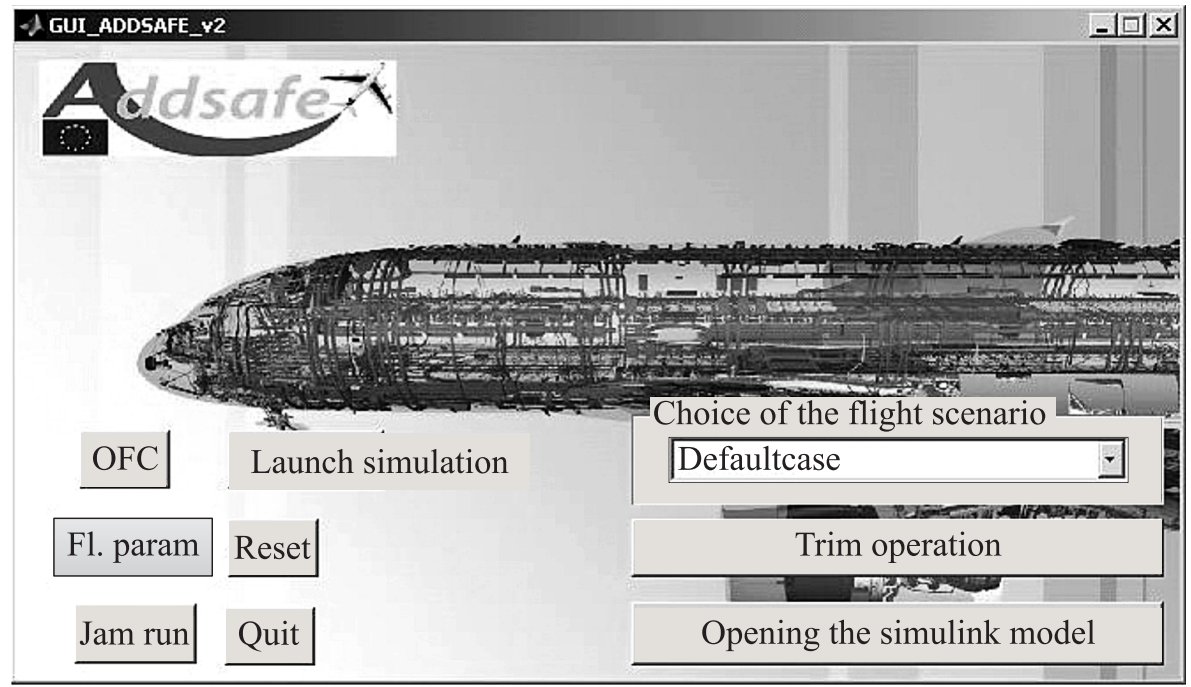

Figure 2 Use of the benchmark through dedicated GUI

in particular, for FDD studies (see section 4). For more handling qualities oriented project, only simple model can be considered like, for instance, first- or second-order transfer functions.

Dedicated GUIs facilitate the use of the benchmark (flight scenario selection, trimming, etc.) and allow high-level tuning of the fault scenario simulation (Fig. 2).

This benchmark has been assessed by comparison with a very high-fidelity and nonexportable in-house simulator. Open-loop as well as closed-loop validations have been performed to compare control surface positions or aerodynamic coefficients without or with solicitations (e.g., $N_{z}$ stick input, rudder pedal input, combined lateral/longitudinal solicitations, etc.). Two examples are depicted below: first, a basic longitudinal solicitation is simulated as a double 10 degree input on the side-stick $\left(+10^{\circ}\right.$, then $\left.-10^{\circ}\right)$. The aerodynamic coefficients (dimensionless quantity) computed on the open-loop model are displayed in Fig. 3. All coefficient behaviors (model outputs) are very close to the reference values shown with dashed curves. Only $C_{M}$ coefficient presents a transient divergence around $30 \mathrm{~s}$ but with no significant consequence on the aircraft behavior. The second example represents a more complex simulation involving a protection law and speed protection (the aircraft speed must remain inside a predetermined envelope for any pilot inputs and wind perturbations). Figure 4 represents the longitudinal parameters behavior during a manoeuvre triggering the speed protection. The model simulation is very close to the reference behavior. 

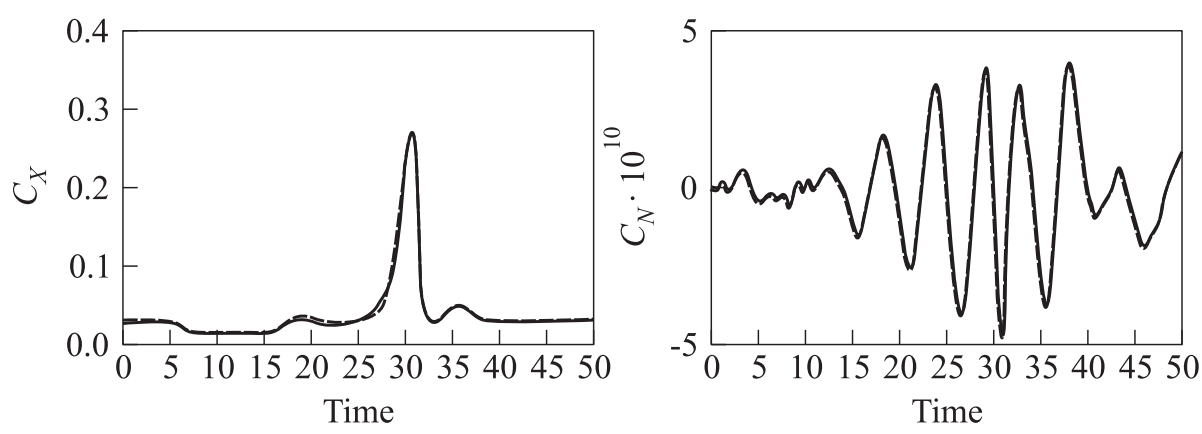

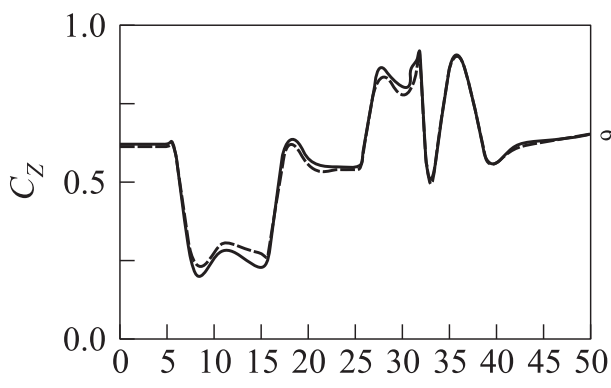

Time

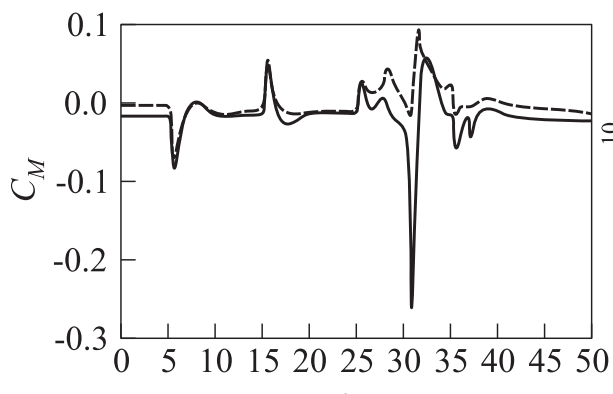

Time
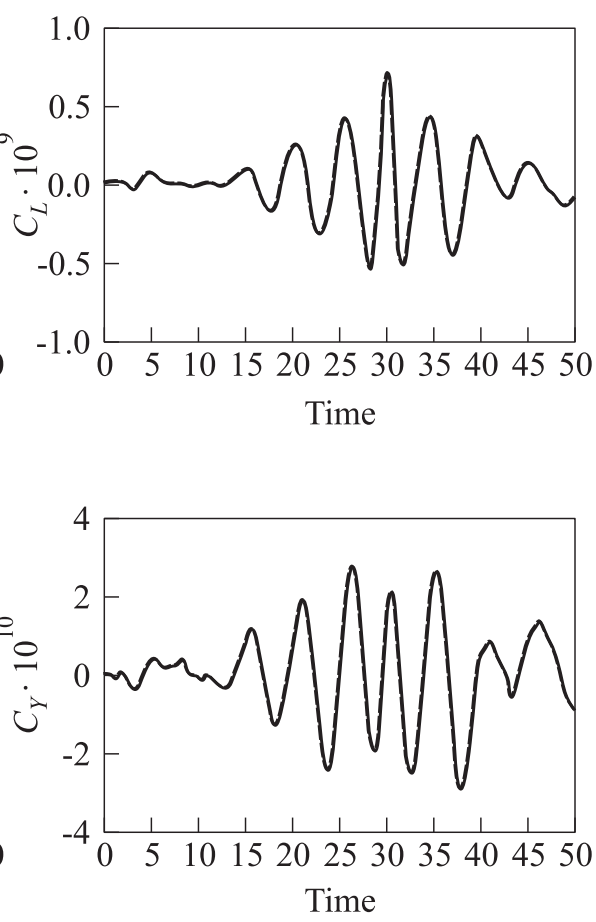

Figure 3 Aerodynamic coefficients comparison on a longitudinal solicitation (solid curves - reference values) 

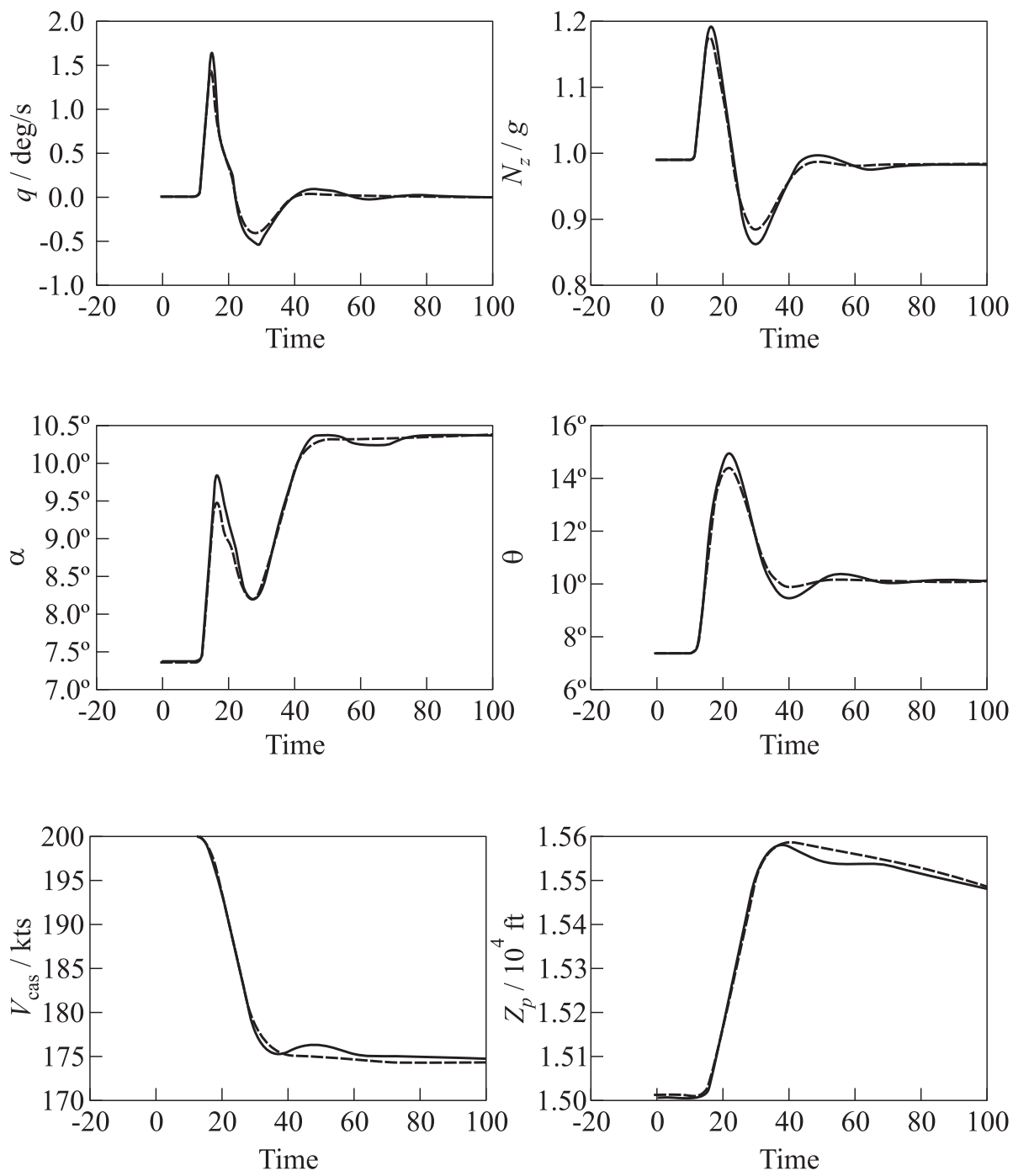

Figure 4 Longitudinal parameters behavior comparison during a speed protection simulation (solid curves - reference values) 


\section{BENCHMARK USE FOR FLIGHT CONTROL LAW CLEARANCE}

The previously described benchmark was first developed for FCL clearance within the framework of the COFCLUO European project [4]. The design of architecture of the Airbus FCL is based on the following two-step requirement:

(1) in the usual operating domain, control laws shall provide an instinctive piloting with the same behavior as a conventional aircraft, as well as an accurate and comfortable control, i. e., stability and manoeuvrability, homogeneous aircraft response with respect to pilot inputs in the whole normal flight envelope, compensation of aircraft configuration changes, minimization of turbulence effect on the flight path and the bank angle, balanced effort in pitch and roll, and safe behavior in the case of engine asymmetry detection; and

(2) in extreme situations, FCL shall provide protections in order to remain in the safe operating domain and reduce the risk of overcontrolling/overstressing the aircraft while at the same time, giving the highest authority to the pilot in order to achieve best possible aircraft performances, e.g., for avoidance manoeuvres.

This leads to two flight envelopes as illustrated in Fig. 5. The normal flight envelope inside which the normal laws are activated and the peripheral flight envelope inside which a set of protection laws ensures both full authority and safety to the pilot.

Linear tools or extensions (LFT - linear fractional transformation, IQC integral quadratic constraint) were used to assess performances of FCL in the normal flight envelope. Use of the benchmark was focussed on the validation of full-flight domain protections in the peripheral domain. Since we are dealing with extreme situations, we are facing high aerodynamic angles, dynamic pilot inputs, and actuator orders. Then, there is a strong need for a high fidelity benchmark. It thus requires a good representativeness even in the nonlinear domain. Those nonlinearities affect the flight mechanics through description of aerodynamic coefficients for high angle of attack or sideslip angle. They are also due to flight control system itself through use of saturations, dead zone, $\min / \max$ operators, etc.

Once the simulation model is as close as possible to the original aircraft to be validated, clearance method can be applied. In the frame of the COFCLUO project, one of the most promising solutions developed is based on use of optimization methods coupled with simulations (Fig. 6).

For utilizing optimization methods, the clearance problem has to be expressed as a scalar objective function $c(p, d)$, named criteria, with optimization parameters $p$ that are uncertain or varying during operation (e.g., inertia, speed, alti- 


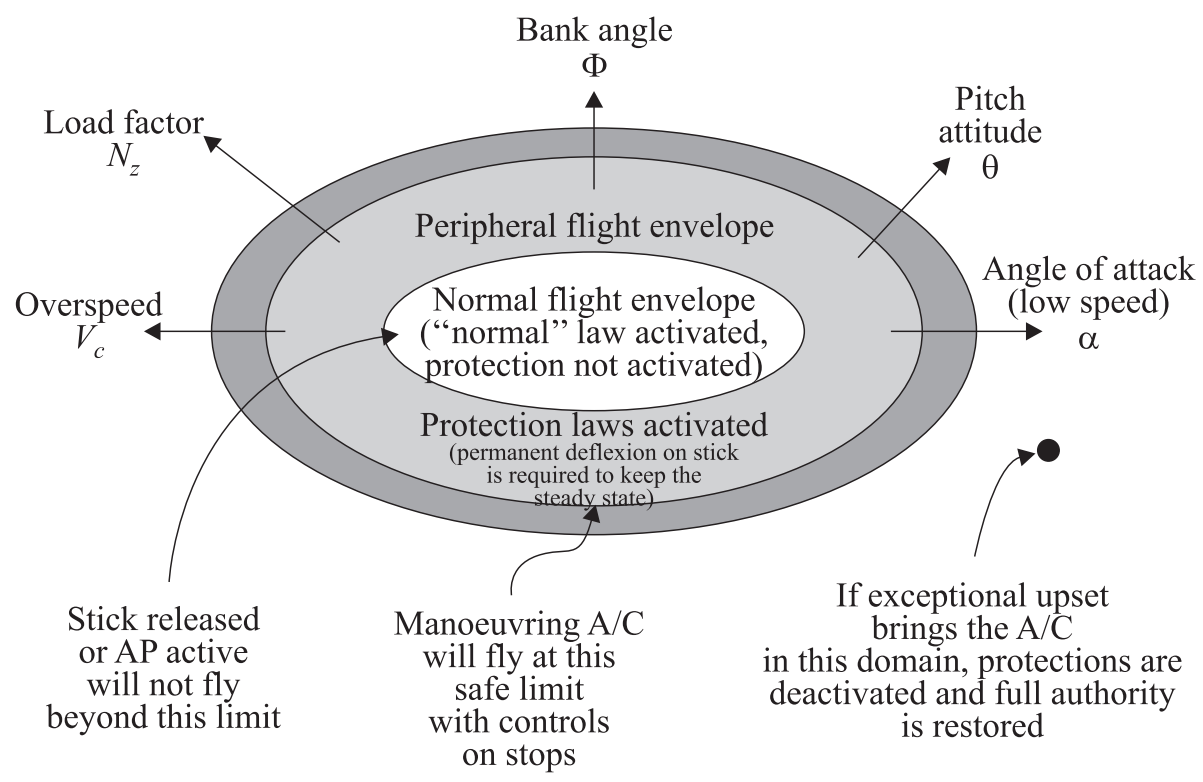

Figure 5 Flight domain definition (A/C stands for aircraft, AP for autopilot)

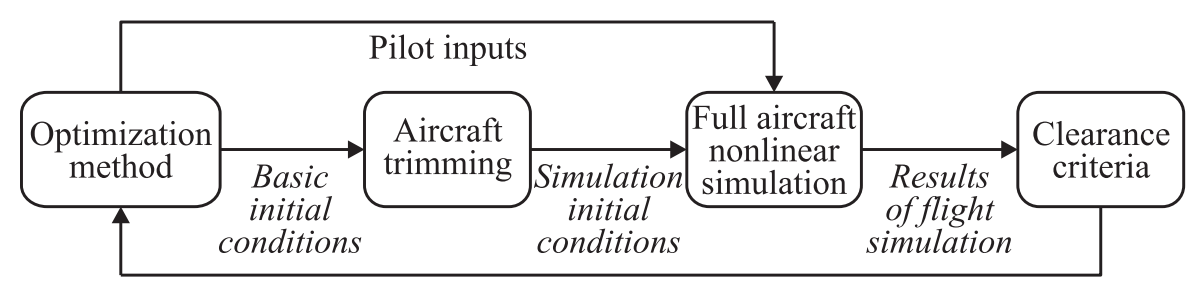

Figure 6 Optimization process

tude, wind conditions, pilot inputs, etc.) and discrete conditions $d$ (e.g., aircraft configuration, landing gear settings, etc.). The components of $p$ are assumed to be bounded and continuously varying over known set $P$. The clearance problem can now be formulated as a minimization problem. Let $c_{0}(d)$ be a lower acceptable value of $c$, then $c_{\min }(d)=\min (c(p, d)), p \in P$, is a measure for the clearance performance. Let assume that the clearance requirement is fulfilled for condition $d$ if $c_{\min }(d) \geq c_{0}(d)$; otherwise, the criterion is not cleared.

Clearance is a long process that uses different means from desktop simulation, in the early stage, to flight tests in the very last phase. Actual early validation is based on a gridding or Monte Carlo simulation approach. It is useful to map performances over the operating domain but it has a poor worst case detection 
rate. It thus can lead to late discovering of problems in the validation phase when they occur in a really tiny region. This issue is solved by the use of optimization as a complement to the previous methods (they are not competing). It should lead to early discovery of FCL weaknesses and, thus, save time and money.

\section{BENCHMARK USE FOR FAULT DETECTION AND DIAGNOSIS}

A consortium of European industrial partners, research establishments, and universities has been established with funding from the European Union 7th Framework Program (EU-FP7) to address the challenge arising from the development of the future "sustainable" aircraft (More Affordable, Smarter, Cleaner, and Quieter). The project is termed ADDSAFE [5]. Highlighting the link between aircraft sustainability and fault detection, it can be demonstrated that improving the fault diagnosis performance in flight control systems allows to optimize the aircraft structural design (resulting in weight saving), which, in turn, helps improve aircraft performance and to decrease its environmental footprint (e.g., fuel consumption and noise). Concretely, if the minimum detectable failure amplitude can be decreased, the aircraft structural design can be improved and the aircraft made lighter. So, the overall aim of the project is to research and develop model-based FDD methods for aircraft flight control system fault detection, predominantly sensor and actuator malfunctions.

The importance of the studies performed within ADDSAFE arises due to the industrial representativeness of the benchmark described in this paper, i. e., the aircraft model, and fault problematic. Moreover, the final goal of the project is to validate the more promising designs in the actual Airbus flight control system verification and validation (V\&V) setup: from high-fidelity simulation models to the Iron Bird, and including real aircraft actuator rigs — which will ensure industry-wide acceptance of the results. From a technological and scientific perspective, the main objectives of the project are:

- identification of a set of guidelines for aircraft guidance and control FDD design and analysis;

- improved FDD methods and understanding of their applicability to aircraft FDD;

- a step towards a $\mathrm{V} \& \mathrm{~V}$ process for aircraft advanced diagnostic systems; and

- demonstration of the most promising model-based FDD designs on industrial state-of-the-art flight simulation platform. 

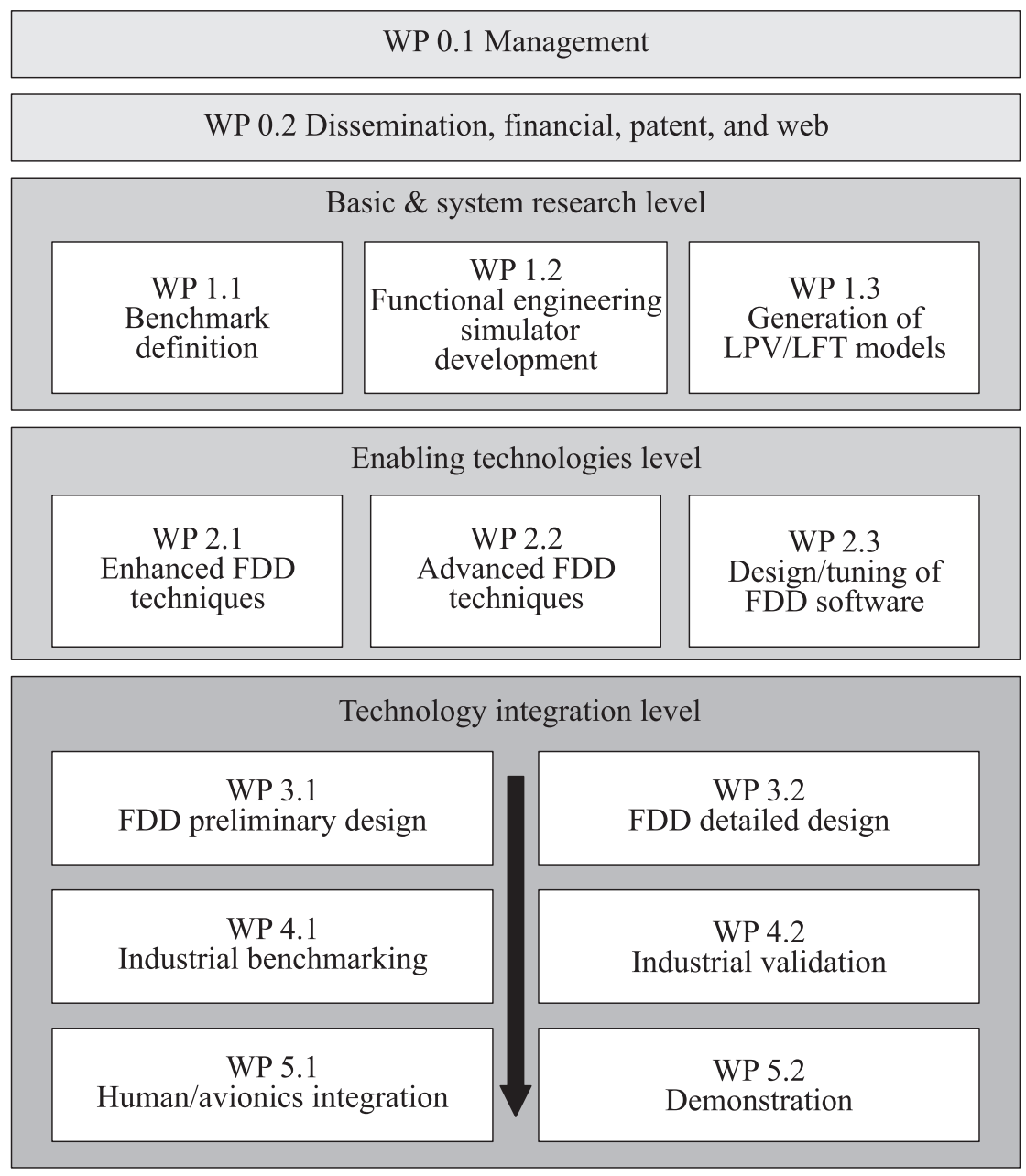

Figure 7 ADDSAFE work package structure (LPV — linear parameter varying)

To achieve these objectives, the project is divided in 6 main work packages (from WP0 to WP5) decomposed into a total of 14 technical work packages. Figure 7 illustrates the link between the technical work packages and the project objectives fashioned along the lines of the Advisory Council for Aeronautics Research in Europe (ACARE) strategic research agenda cycle: Basic and System Research Level, Enabling Technologies Level, and Technology Integration Level.

The FDD benchmark (WP1.1) to be addressed in ADDSAFE includes the aircraft model development as well as the fault scenario definition. The whole 
benchmark definition will serve as a design specification for WP3 (applications of FDD designs) and for the industrial assessment in WP4. Three kinds of scenarios have been defined [5] for covering a wide range of possible sensor and actuator faults related to structural design objectives and aircraft performance, highlighting the impact on the aircraft sustainability.

Compared to the benchmark use for FCL clearance, for FDD studies, it has been modified for more actuator and sensor model fidelity and to open up the possibility to inject representative faults. Actuator modeling covers the control loop and allows simulating fault at a component level like, for example, on flight control computer input/output which is impossible with a simple transfer function. Fault propagation through the control loop also allows to reproduce nonlinearity and to synthesize a more representative fault. For example, for oscillatory failure case [6], rather than simulating a pure sinusoid on the control surface position, a sinusoid injected at a component level will lead to a more representative oscillation of the control surface, transformed by the nonlinearity. Similarly, more accurate sensor modeling (clinometric, inertial, etc.) allows more realistic simulation of a wide range of fault types.

The benchmark closed-loop simulation environment and representativeness allow assessment on the whole flight domain considering a wide class of pilot inputs and perturbations which is crucial for FDD design robustness validation. Moreover, the embedded flight control laws guarantee an aircraft reaction after failure occurrence, and so, a more realistic aircraft behavior. It also represents a step towards industrial assessment before test bench and flight simulator validation.

\section{CONCLUDING REMARKS}

Summarizing, this paper is dedicated to a general description of a generic civil aircraft model developed by Airbus for advanced research on flight control system. Clearly, the level of modeling can be adapted to the kind of study performed. For example, basic actuator models are satisfactory for handling qualities oriented works whereas more detailed models are needed for projects dedicated to system component FDD. This aircraft model is well adapted to a wide range of flight control topics.

The aerodynamic database model is quite difficult to obtain. Some improvement could be made as some nonrealistic behaviors have been noticed on some particular points of the flight domain. However, this does not compromise the whole aircraft model representativeness. This aerodynamic modeling will be the topic of future works as well as a more and more detailed sensor and actuator modeling for extended FDD studies to system component fault isolation. 


\section{ACKNOWLEDGMENTS}

This work was performed in the framework of the European ADDSAFE Project: Grant agreement No. FP7-233815.

\section{REFERENCES}

1. Goupil, P. 2011. AIRBUS state of the art and practices on FDI and FTC in flight control system. Control Eng. Practice 19:524-39. DOI information: 10.1016/j.conengprac.2010.12.009.

2. Edwards, C., T. Lombaerts, and H. Smaili, eds. 2010. Fault tolerant flight control: A benchmark challenge. Lecture notes in control and information sciences ser. Berlin-Heidelberg: Springer-Verlag. 399.

3. Marcos, A., and G.J. Balas. 2003. A Boeing 747-100/200 aircraft fault tolerant and fault diagnosis benchmark. Technical Report AEM-UoM-2003-1. Minnesota: University of Minnesota.

4. Varga, A., A. Hansson, and G. Puyou. 2012. Optimization based clearance of flight control laws: A civil aircraft application. Lecture notes in control and information sciences ser. Springer-Verlag. 416.

5. Goupil, P., and A. Marcos. 2011. Advanced diagnosis for sustainable flight guidance and control: The European ADDSAFE project. SAE 2011 AeroTech Congress and Exhibition. Toulouse, France.

6. Goupil, P. 2010. Oscillatory failure case detection in the A380 Electrical Flight Control System by analytical redundancy. Control Eng. Practice 18:1110-19. DOI:10.1016/j.conengprac.2009.04.003. 